\title{
TOPICAL ANTI-INFLAMMATORY EFFECTS OF AF 2071: A COMPARATIVE STUDY WITH BENDAZAC AND CORTICOSTEROIDS, BOTH ALONE AND ASSOCIATED
}

\author{
R. LISCIANI, G. DE FEO, V. CIOLI and S. PUTZOLU \\ F. Angelini Research Institute, Viale Amelia, 70-00181, Rome, Italy
}

Accepted November 13, 1974

\begin{abstract}
Bendazac has anti-inflammatory effects which are different from or complementary to those of corticosteroids; therefore, it has been associated with hydrocortisone or dexamethasone, in the attempt to obtain a dermatological preparation with a wide range of therapeutic applications. Results in laboratory animals were discouraging, in that a diminished activity was observed. On the other hand, bendacort or (pregn-4ene 3, 20 dione 11 $\beta, 17 \alpha, 21$ trihydroxy, 21 ester with acetic acid [(1-phenylmethyl-1 H-indazol-3-yl)-oxy], inhibited both types of inflammatory responses which are sensitive either to bendazac or corticosteroids. Moreover, in acetic acid-induced erythema, which is sensitive to both types of drugs, AF 2071 had stronger effects than those produced by bendazac or corticosteroids. Therapeutic and theoretical implications of these results are discussed.
\end{abstract}

Bendazac or 1-benzyl-indazole-3 oxyacetic acid is a topical anti-inflammatory drug which has been extensively studied both in laboratory tests and in humans (1). It has been demonstrated to have anti-inflammatory effects which are different and often complementary to those of hydrocortisone. In fact, whereas hydrocortisone is particularly active on responses involving granulona or wound healing where defence mechanisms prevail, bendazac acts preferentially on responses with a necrotic evolution where tissue damage predominates; only in the case of erythema due to chemical agents, such as acetic acid, does the activity of the two drugs tend to overlap (2, 3; Sato et al., personal communication). Since in the most common inflammatory conditions tissue damage and defence mechanisms are closely related $(4,5)$, the above results suggested the study of a preparation combining the properties of corticosteroids with those of bendazac.

In this connection, we took into consideration both an association of bendazac with corticosteroids as well as AF 2071 or (pregn-4 ene 3, 20 dione 11 $171 \alpha, 21$ trihydroxy. 21 ester with acetic acid [(1-phenylmethyl-1/1-indazol-3-y])-oxy], a compound synthetized in our laboratorics by means of esterification of bendazac with hydrocortisone. These were first studied using an inflammatory response sensitive to both bendazac and steroids, in order to obtain a quantitative evaluation of activity. In a second phase, the preparations worthy of further investigation were studied on experimental models of inflammation which allowed the spectrum of action to be determined.

\section{MATERIALS AND METHODS}

In all experiments, Long Evans rats of both sexes weighing between 140 and $180 \mathrm{~g}$ were used. The results obtained in males and females were grouped together since 
no significant differences were observed. For applications to the skin, the products were blended in a standard oil-in-water emulsion. Some experiments with AF 2071 have been duplicated using different formulations and this has not modified the effects of the drug.

The following drugs were used: bendazac, dexamethasone alcohol, hydrocortisone acetate, AF 2071 (synthetized by A.C.R. Angelini Francesco, Rome, Italy).

The results obtained were elaborated using the Student's " $t$ " method and covariance analysis. In the case of acetic acid-induced erythema and heat-induced burns the original values were transformed into a percentage of the highest response according to a previously described method (2).

With acetic acid-induced erythema we referred to a method previously used in mice (3). Throughout the entire experiment the animals were kept under urethane anaesthesia $(1.5 \mathrm{~g} / \mathrm{kg}$ i.p.). A disc of filter paper, $7 \mathrm{~mm}$ in diameter, was soaked with glacial acetic acid and placed on the previously shaven abdomen of the animals. After $15 \mathrm{sec}$ the disc was removed and the excess acetic acid dried. One min later, $0.5 \mathrm{ml}$ of the creams under study were applied locally, and one hr later the animals were injected i.v. with $1 \mathrm{ml}$ of $0.5 \%$ Evans blue in physiological saline. After $90 \mathrm{~min}$ the animals were sacrificed by means of a blow on the back of the neck and the skin of the treated area was removed and inserted between two glass slides. The intensity of the blue staining due to the accumulation of Evans blue in the irritated skin area was estimated using a Joyce \& Loebl mod. II photodensitometer.

A slightly altered version of the method described by Lisciani et al. (3) was adopted for the heat-induced burns. The rats were kept under urethane anaesthesia ( $1.5 \mathrm{~g} / \mathrm{kg}$ i.p.) throughout the entire experiment. Burns were produced by placing a test tube (weighing $50 \mathrm{~g}$ ) in which water was circulating at a temp. of $70^{\circ} \mathrm{C}$ on the previously shaven abdomen of the animals for $15 \mathrm{sec}$. One min later $0.5 \mathrm{ml}$ of the creams under study were applied locally; one hr later the animals were injected i.v. with $1 \mathrm{ml}$ of an $0.5 \%$ Evans blue solution in saline. Two hr later the rats were sacrificed by means of a blow on the back of the neck and the skin of the treated area was removed and inserted between two glass slides. The intensity of the blue staining due to the accumulation of Evans blue was evaluated using the methods alteady described for acetic acid-induced erythema.

The method adopted by Selye (6) was used for urea-induced necrosis of the skin. Two $\mathrm{ml} / \mathrm{rat}$ of a solution containing $20 \%$ urea in $0.5 \%$ methylcellulose was injected s.c. into the animals" flank previously shaven; immediately afterwards, $10 \mathrm{mg} / \mathrm{kg}$ of serotonincreatinine sulphate was injected i.v. The products under study were suspended in the solution of urea and methylcellulose so as to obtain a tinal concentration of $10^{-2} \mathrm{M}$. The degree of necrosis was evaluated each day for a total of 5 consecutive days starting $24 \mathrm{hr}$ after the urea injection. The following scoring system was employed:

0 : no difference from normal skin

1: a small area of necrosis in the centre of the urea injection site

2: areas of necrosis scattered over the urea injection site 
3: wide area of necrosis extending over the entire urea injection site

4: wide area of necrosis which also involves the deeper layers of the dermis, accompanied by a loss of tissue.

Cicatrization was investigated by removing about $1 \mathrm{~cm}^{2}$ of skin from the back which had been previously shaven. Two ml of the creams under study were applied daily on the wound for a total of 10 days. During the first $24 \mathrm{hr}$ an occlusive dressing was applied to allow a better contact with the skin. The size of the wound was measured every second day throughout the entire experiment.

Cotton pellet granuloma was obtained according to the method of Meier et al. (7). Two sterile cotton pellets weighing $70-75 \mathrm{mg}$ were implanted symmetrically into the back of each rat; one sterile pellet was soaked with $0.5 \mathrm{ml}$ of an ethanol solution of the products under study; the other with the solvent alone. The cotton pellets were dried $\left(40^{\circ} \mathrm{C}\right.$ for $24 \mathrm{hr}$ ) before the implantation. Three days later the animals were sacrificed and the weights of both the wet and the dry granuloma were determined.

\section{RESULTS}

Effects of various concentrations of hydrocortisone, dexamethasone and bendazac in acetic acid-induced erythema are reported in Fig. 1. The dilution factors used do not allow dose/effect curves to be obtained; in fact, we pass from concentrations devoid of significant effects to concentrations with ceiling effect. The latter is manifested, with all three products under study, at inhibition values slightly over $30 \%$.

The minimal active doses of the three drugs were used for studying a possible antagonism between bendazac and the two corticosteroids in the acetic acid-induced erythema test. Results are given in Table 1.

The association of bendazac with the two corticosteroids gives rise to antagonism: in fact, when active concentrations of the drugs were associated, significant effects were no longer shown. Results obtained by studying AF 2071 in acetic acid-induced erythema are shown in Table 2; hydrocortisone and bendazac at equimolecular concentrations were used as reference drugs. AF 2071 produces an inhibition which is almost

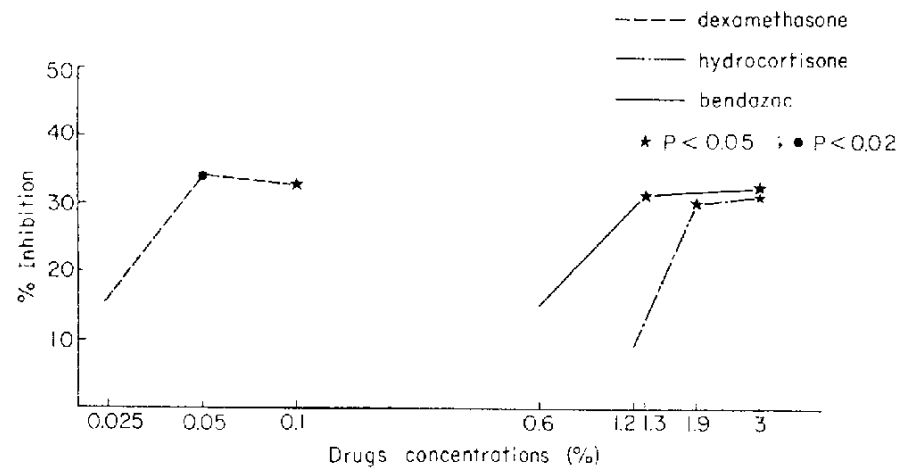

FIG. 1. Effects of dexamethasone, hydrocortisone and bendazac on acetic acidinduced erythema. At least 10 rats were used for each determination. 
TABLE 1. Effects of bendazac, dexamethasone and hydrocortisone, both alone and associated, on acetic acid-induced erythema.

\begin{tabular}{lccc}
\hline \multicolumn{1}{c}{ Treatment } & $\begin{array}{c}\text { No. of } \\
\text { rats }\end{array}$ & $\begin{array}{c}\text { Blue area } \\
\pm \mathrm{SE}\end{array}$ & $\begin{array}{c}\% \text { inhibition as compared } \\
\text { to controls }\end{array}$ \\
Excipient & 25 & $53 \pm 5.8$ & \\
Bendazac 1.3\% & 23 & $37 \pm 4.5$ & $30^{\mathrm{a}}$ \\
Hydrocortisone $1.9 \%$ & 23 & $37 \pm 5.0$ & $30^{\mathrm{a}}$ \\
$\begin{array}{l}\text { Hydrocortisone } 1.9 \% \\
\text { + Bendazac } 1.3 \%\end{array}$ & 22 & $45 \pm 4.6$ & 15 \\
Excipient & 15 & $60 \pm 5.5$ & \\
Bendazac $1.3 \%$ & 12 & $43 \pm 5.1$ & $28^{\mathrm{a}}$ \\
Dexamethasone $0.05 \%$ & 16 & $39 \pm 5.6$ & $35^{\mathrm{b}}$ \\
$\begin{array}{l}\text { Dexamethasone 0.05\% } \\
\text { t-Bendazac } 1.3 \%\end{array}$ & 17 & $50 \pm 5.1$ & 17 \\
\hline
\end{tabular}

a $\mathrm{P}<0.05 ; \quad$ b $\mathrm{P}<0.02$

TABLF 2. Effects of hydrocortisone, bendazac and AF 2071 on acetic acid-induced erythema.

\begin{tabular}{lccc}
\hline \multicolumn{1}{c}{ Treatment } & $\begin{array}{c}\text { No. of } \\
\text { rats }\end{array}$ & $\begin{array}{c}\text { Blue area } \\
\pm \mathrm{SE}\end{array}$ & $\begin{array}{c}\% \text { inhibition as compared } \\
\text { to controls }\end{array}$ \\
Excipient & 25 & $53 \pm 5.8$ & \\
Hydrocortisone $1.9 \%$ & 22 & $37 \pm 5.0$ & $30^{\mathrm{a}}$ \\
Bendazac $1.3 \%$ & 23 & $37 \div 4.5$ & $30^{\mathrm{a}}$ \\
AF 2071 3\% & 22 & $23 \pm 4.7$ & $49^{\circ}$ \\
\hline
\end{tabular}

a $\mathrm{P}<0.05 ; \mathrm{c} \mathrm{P}<0.01$

TABLE 3. Effects of hydrocortisone, bendazac and AF 2071 on heat-induced burns.

\begin{tabular}{lccc} 
Treatment & $\begin{array}{c}\text { No. of } \\
\text { rats }\end{array}$ & $\begin{array}{c}\text { Blue area } \\
\pm \mathrm{SE}\end{array}$ & $\begin{array}{c}\% \text { inhibition as compared } \\
\text { to controls }\end{array}$ \\
Excipient & 24 & $48+5.1$ & \\
Hydrocortisone $3 \%$ & 20 & $49 \pm 6.3$ & 0 \\
Bendazac $1.3 \%$ & 19 & $34 \pm 4.6$ & $29^{\mathrm{a}}$ \\
AF 2071 $3 \%$ & 24 & $34 \pm 4.3$ & $29^{\mathrm{a}}$ \\
\hline
\end{tabular}

a $\mathrm{P}<0.05$

equal to the sum of those displayed by bendazac and hydrocortisone.

Results obtained by studying the effects of AF 2071 on heat-induced burns are reported in Table 3; bendazac and hydrocortisone were used als reference drugs. AF 2071 and bendazac exert, at equimolecular concentrations, effects of equal degree. Hydrocortisone, according to previous results (3), is inactive up to a $3 \%$ concentration.

Results obtained by studying the effects of AF 2071 on skin necrosis according to Selye (6) are summarized in Fig. 2; hydrocortisone and bendazac at equimolecular concentrations were used as reference drugs. Hydrocortisone, in agreement with previous data (2), aggravated the necrotic reaction. Bendazac and AF 2071 showed almost equal protective effects even though those of AF 2071 were delayed as compared to those of bendazac. 


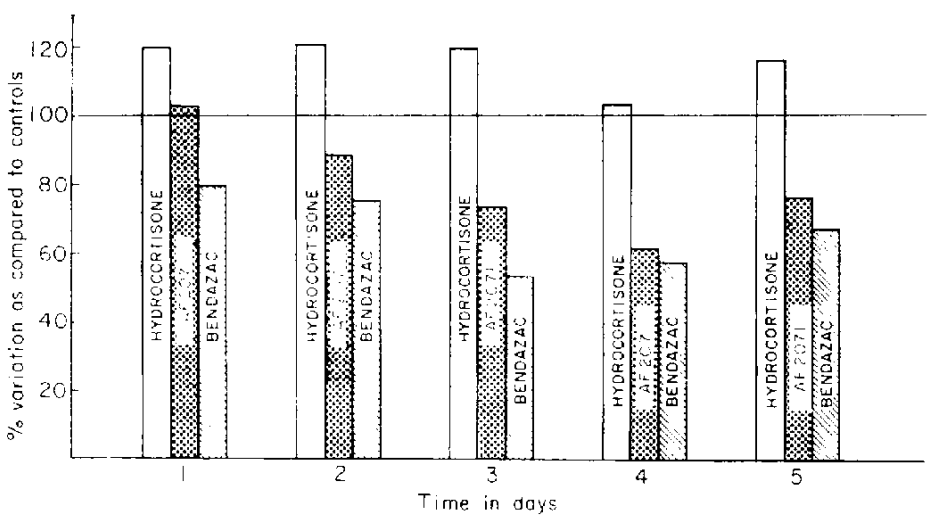

FIG. 2. Effects of hydrocortisone, AF 2071 and bendazac on skin necrosis according to Selye. The mean value of the controls made equal to 100 is represented by the continuous line. A total of 10 rats were used for each determination.

TABL: 4. Effects of bendazac, hydrocortisone and AF 2071 on cotton pellet-induced granuloma.

At least 10 animals were used for each determination.

\begin{tabular}{lccc}
\hline \multicolumn{1}{c}{ Products } & $\begin{array}{c}\text { Concentration } \\
, \text { gg/pellet }\end{array}$ & $\begin{array}{c}\text { Wt. of wet granuloma } \\
\text { in mg } \\
\text { inhibition })\end{array}$ & $\begin{array}{c}\text { Wt. of dry granuloma } \\
\text { in mg } \\
(\% \text { inhibition })\end{array}$ \\
Controls & & 977 & 110 \\
Bendazac & 500 & 1023 & 124 \\
Hydrocortisone & 62 & $839\left(14^{\mathrm{b}}\right)$ & $62\left(44^{\mathrm{d}}\right)$ \\
AF 2071 & 100 & $689\left(29^{\mathrm{d}}\right)$ & $60\left(45^{\mathrm{d}}\right)$ \\
Controls & & 884 & 97 \\
Hydrocortisone & 31 & 918 & $81\left(16^{\mathrm{a}}\right)$ \\
AF 2071 & 50 & $725\left(18^{\mathrm{d}}\right)$ & $60\left(38^{\mathrm{d}}\right)$ \\
\hline
\end{tabular}

b $P<0.02 ;$ a $P<0.05 ;$ d $P<0.001$

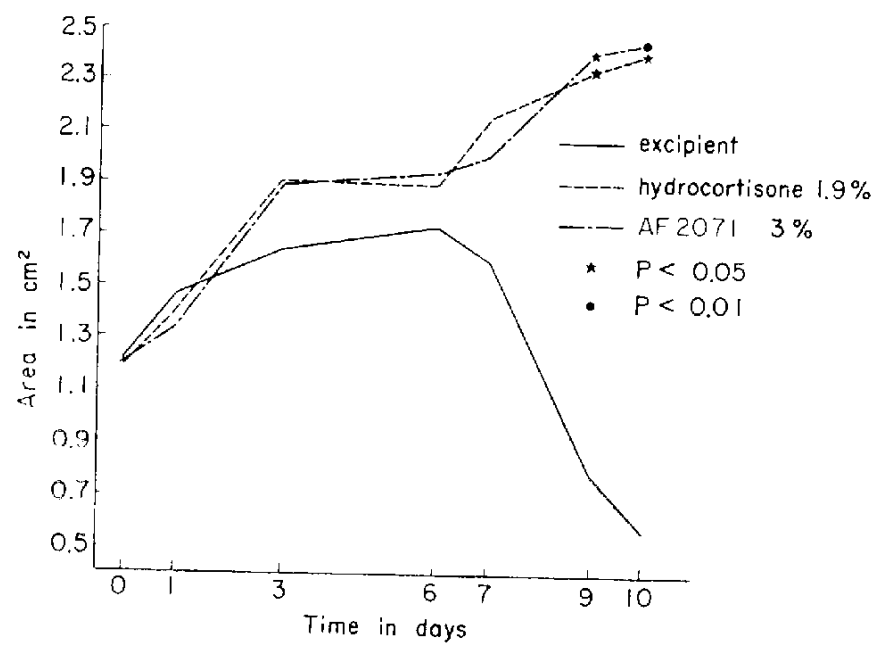

FIC. 3. Effects of hydrocortisone and AF 2071 on wound healing process. A total of 10 rats were used for each determination. 
Results obtained by studying AF 2071 in cotton pellet granuloma are reported in Table 4; hydrocortisone and bendazac were used as reference drugs. Bendazac proved to be inactive up to the concentration of $500 \mu \mathrm{g} /$ cotton pellet. AF 2071 showed an activity equal to that of equimolecular concentrations of hydrocortisone. Both products had a stronger action on the proliferative than on the exudative response, as shown by the greater activity exerted on the dry granuloma than on the wet granuloma.

Fig. 3 shows the results obtained by studying the healing process: bendazac, hydrocortisone and AF 2071 were applied at equimolecular concentrations. Bendazac at a $1.3 \%$ concentration had no effect (data not reported in fig.), whereas both hydrocortisone and AF 2071 delayed the healing process.

\section{DISCUSSION}

Our results show that the association of bendazac with corticosteroids does not satisfy the purpose of this investigation, namely, an anti-inflammatory dermatological preparation endowed with a wider spectrum of action than that of presently available drugs. The association, in fact, showed anti-inflammatory effects inferior to those of bendazac or steroids used alone. The same interaction was manifested with two different corticosteroids, namely hydrocortisone and dexamethasone. Even if the mechanism of this undesired interaction has still not been clarified, it is so marked as to discourage the use of this type of association in therapy. Therefore, we resorted to the study of the ester of bendazac and hydrocortisone, i.e. AF 2071. The main results obtained with AF 2071 have

TABLE 5. Topical effects of AF 2071, hydrocortisone and bendazac on different inflammatory responses.

\begin{tabular}{lccccc}
\hline \multicolumn{1}{c}{ Products } & $\begin{array}{c}\text { Heat-induced } \\
\text { burns }\end{array}$ & $\begin{array}{c}\text { Skin } \\
\text { necrosis }\end{array}$ & $\begin{array}{c}\text { Acetic acid- } \\
\text { induced erythema }\end{array}$ & $\begin{array}{c}\text { Cotton pellet } \\
\text { granuloma }\end{array}$ & Cicatrization \\
AF 2071 & + & + & + & + & + \\
Bendazac & + & + & + & - & - \\
Hydrocortisone & - & - & + & + & + \\
\hline
\end{tabular}

+ positive effect

- no effect

been schematically illustrated in Table 5 where it is compared with bendazac and hydrocortisone. It appears evident that AF 2071 has a wider spectrum of anti-inflammatory effects than bendazac or hydrocortisone alone; this spectrum includes inflammatory responses with a necrotic evolution as well as those in which the reaction of tissue defence predominates, as for example in the healing of wounds.

Besides its spectrum of effects, AF 2071 also differs from bendazac and hydrocortisone quantitatively. With bendazac and hydrocortisone, in the case of acetic acid-induced erythema, relatively moderate inhibition values of about $30 \%$ wcre obtained. In the case of AF 2071, instead, more marked inhibition values were obtained, i.e. around $50 \%$. Since with hydrocortisone and bendazac such inhibition values cannot be obtained even with increased concentrations, the results obtained with AF 2071 can be explained 
by assuming that this drug simultaneously exerts the two different effects characteristic of hydrocortisone and bendazac respectively, i.e. the inhibition of active tissue response and the protection against tissue damage.

On the basis of our results it may be concluded that AF 2071 is a drug which is entirely different from those so far available for the topical treatment of inflammatory conditions of the skin.

\section{REFERENCES}

1) De Gregorio, M. And Fugia, G.C.: Proceedings of International Symposium "New Trends in Skin Therapeutics" Leonardo Ed. Scientifiche (in press).

2) Silyestrini, B., Cloli, V. and Burberi, S.: Arzneim.-Forsch., 19, 30 (1969).

3) Lisciani, R., Scorza Barcellona, P. and Silvestrin, B. Japan. J. Pharmacol, 21, 30 (1969).

4) GozsY, B. AND KATO, L.: Balancing mechanisms in acute inflammation, Edited by Institute of Microbiology and Hygiene of Montreal University, Monograph n. 5, p. 16, Montreal (1970).

5) Willovghiy, D.A.: The inflammatory process, Edited by Zweifach, B.W., Grant, L., MCCluskey, R.T., p. 303, Academic Press, New York and London (1973).

6) Selye, H.: Science, 152, 1371 (1966).

7) Meier, R., Schulfr, W. and Desaulles, P.: Experientia, 6, 469 (1950). 